\title{
SAMPLING SPHERICAL FINITE RATE OF INNOVATION SIGNALS
}

\author{
Ivan Dokmanić
}

\author{
School of Computer and Communication Sciences \\ Ecole Polytechnique Fédérale de Lausanne (EPFL) \\ CH-1015 Lausanne, Switzerland \\ ivan.dokmanic@epfl.ch
}

\author{
Yие M. Lu
}

\begin{abstract}
We propose a sampling scheme that can perfectly reconstruct a collection of spikes on the sphere from samples of their lowpassfiltered observations. The proposed algorithm can reconstruct $K$ spikes from $(K+\sqrt{K})^{2}$ spatial samples, thus improving over previously known FRI sampling schemes on the sphere by a factor of up to four. Further, we show how multiple sound source localization (SSL) by a spherical microphone array can be transformed into a spherical FRI sampling problem. We certify the effectiveness of the proposed algorithm by using it to solve the SSL problem.
\end{abstract}

Index Terms - Sphere, finite rate of innovation, sampling, sparsity, sound source localization

\section{INTRODUCTION}

Spherical signals appear frequently in a number of distinct domains. Examples are signals defined on Earth's surface [1-3], signals from space measured on Earth $[4,5]$, or spherical microphone arrays that output a time-varying signal supported on a sphere [6,7].

Often, it is realistic to model the signal of interest as a weighted sum of localized spikes. In the limit, spikes become Dirac delta functions. For example, many acoustic sources are well-aproximated by point sources. The directional distribution of multiple sources is then a finite collection of spikes. Stars in the sky observed from Earth are angular spikes, and so are plume sources on Earth. Collections of spikes are signals with a finite rate of innovation (FRI). In the above examples, we observe the spikes through sampling kernels (for example point spread functions and Green's functions). The essential property of kernels is that they are approximately bandlimited. The resulting spherical signal is then bandlimited, as it is a superposition of rotations of bandlimited kernels.

An archetypal FRI signal is a weighted sum of Dirac delta functions [8]; many other FRI signals are derived by processing sums of Diracs. Integrating Diracs yields polynomial splines in 1D, and bilevel polygons in 2D. The authors in [8] discuss periodic streams of Diracs and related signals. In particular, they show that the $T$ periodic signal $x(t)=\sum_{i \in \mathbb{Z}} \sum_{k=1}^{K} c_{k} \delta\left(t-t_{k}-i T\right)$ with the rate of innovation of $2 K / T$ can be reconstructed from $2 K+1$ samples. A more detailed discussion of the performance in the noisy case is given in [9] and [10].

In higher dimensions, unlike in the 1D case, the signals are not sampled at the rate of innovation $[11,12]$. Nevertheless, the term FRI is still used, as the algorithms allow to directly compute the degrees

I. Dokmanić was supported by an ERC Advanced Grant-Support for Frontier Research-SPARSAM Nr: 247006, and a Google PhD Fellowship. Y. M. Lu was supported in part by the U.S. National Science Foundation under Grant CCF-1319140. of freedom. In $2 \mathrm{D}$, due to the properties of zeros of multivariate polynomials, the required number of samples is $O\left(K^{2}\right)$.

In this paper, we propose an algorithm to perfectly reconstruct collections of spikes from their lowpass-filtered observations. Our algorithm efficiently reconstructs $K$ spikes when the bandwidth of the lowpass filter is at least $K+\sqrt{K}$. Using recent results on efficient computation of the spherical Fourier transform $[13,14]$, this implies that we need $(K+\sqrt{K})^{2}$ spherical samples to reconstruct the $K$ spikes.

In [15], the authors proposed a spherical FRI sampling scheme requiring $(2 K)^{2}$ samples to reconstruct a distribution of $K$ Diracs. They further notice that if only $3 K$ spectral bins are active, they can use the optimal number of samples. Sampling at this critical rate relies on the assumption that we can apply arbitrary spectral filters before sampling the signal. But complex spectral filtering is generally not possible by operating on $3 K$ spatial samples; we must compute the spectrum before applying the filter.

This observation reveals a semantic difference between spatial (multi-dimensional) FRI sampling and FRI sampling in time. In the latter case, the signal is not bandlimited, yet we can reconstruct it from the critical number of samples because we can perform analog anti-aliasing filtering. On the other hand, in spatial sampling, the signal should be bandlimited before sampling. The value of spatial or spherical FRI sampling lies in direct, efficient extraction of signal parameters from a small number of samples.

In Section 2 we list the necessary results from harmonic analysis on the sphere. Section 3 develops the main result, and in Section 4 we describe the application of the result to sound source localization. Due to space limitations, we defer the proofs of lemmas and theorems to a forthcoming longer paper [16].

\section{HARMONIC ANALYSIS ON THE SPHERE}

We briefly recall several definitions related to spherical harmonics. The 2-sphere is defined as a locus of points in $\mathbb{R}^{3}$ with unit norm, $\mathbb{S}^{2}=\left\{\mathbf{x} \in \mathbb{R}^{3} \mid \mathbf{x}^{T} \mathbf{x}=1\right\}$. Spherical harmonics form a natural (orthonormal) Fourier basis for the Hilbert space of square integrable functions on the sphere. They are defined as $[17]^{1}$

$$
Y_{\ell}^{m}(\theta, \phi)=N_{\ell}^{m} P_{\ell}^{m}(\cos \theta) e^{j m \phi},
$$

where $N_{\ell}^{m}$ is the normalization constant, and $P_{\ell}^{m}$ are the associated Legendre polynomials,

\footnotetext{
${ }^{1}$ Spherical harmonic order $m$ is conventionally displayed as a superscript. We will keep this convention for the associated Legendre polynomials $P_{\ell}^{|m|}$, spherical harmonics $Y_{\ell}^{m}$, normalization constants $N_{\ell}^{m}$, and the spherical Fourier coefficients $f_{\ell}^{m}$. It should not be confused with powers as in $x^{\ell}$.
} 


$$
P_{\ell}^{m}(x)=(-1)^{m}\left(1-x^{2}\right)^{m / 2} \frac{d^{m}}{d x^{m}}\left(P_{\ell}(x)\right), \text { for } m \geq 0 .
$$

$P_{\ell}(x)$ is the Legendre polynomial of degree $\ell$.

Any square integrable function on the sphere, $f \in L^{2}\left(\mathbb{S}^{2}\right)$, can be expanded in the spherical harmonic basis,

$$
f(\theta, \phi)=\sum_{\ell=0}^{\infty} \sum_{|m| \leq \ell} f_{\ell}^{m} Y_{\ell}^{m}(\theta, \phi) .
$$

The expansion coefficients are computed as $f_{\ell}^{m}=\left\langle f, Y_{\ell}^{m}\right\rangle=$ $\int_{\mathbb{S}^{2}} f(\omega) Y_{\ell}^{m}(\omega) \mathrm{d} \omega$, where $\mathrm{d} \omega=\sin (\theta) \mathrm{d} \theta \mathrm{d} \phi$ is the usual rotationally invariant measure on the sphere. The coefficients $\left(f_{\ell}^{m}\right)_{(l, m) \in \mathcal{I}}$ form a countable set supported on a triangle of indices,

$$
\mathcal{I}=\left\{(\ell, m) \in \mathbb{Z}^{2}|\ell \geq 0,| m \mid \leq \ell\right\} .
$$

We say that $f$ is bandlimited with bandwidth $L$ if $L$ is the smallest integer such that $f_{\ell}^{m} \equiv 0$ for $\ell \geq L$.

We define the spherical Dirac delta function [18] so that it satisfies the sifting property, $\int_{\mathbb{S}^{2}} \delta\left(\omega ; \omega_{0}\right) f(\omega) \mathrm{d} \omega=f\left(\omega_{0}\right), \forall \omega_{0} \in \mathbb{S}^{2}$ and "nice" $f$. To address the case of a weighted sum of rotations of a known function, we use (non-commutative) spherical convolution, thereby reducing it to a sum of Diracs. Let $g=f * h$. Then [17]

$$
g_{\ell}^{m}=(2 \ell+1)^{-1 / 2} f_{\ell}^{m} h_{\ell}^{0} .
$$

\section{SAMPLING SPHERICAL FRI SIGNALS}

We aim to reconstruct weighted sums of Diracs, or more generally, sums of rotations of a known kernel. The kernel is typically predesigned (e.g the point spread function), and we cannot change it. The signal of interest is given as

$$
f(\theta, \phi)=\sum_{k=1}^{K} \alpha_{k} \delta\left(\theta, \phi ; \theta_{k}, \phi_{k}\right),
$$

where $\alpha_{k} \in \mathbb{C}$. The Fourier transform of $f$ (in the sense of distributions) is

$$
f_{\ell}^{m}=\sum_{k=1}^{K} \alpha_{k} \overline{Y_{\ell}^{m}\left(\theta_{k}, \phi_{k}\right)} .
$$

\subsection{Finite Rate of Innovation Sampling}

Using the definition of associated Legendre polynomials (2), we rewrite the spherical harmonics (1) as

$$
Y_{\ell}^{m}(\theta, \phi)=N_{\ell}^{m}(\sin \theta)^{|m|}\left[\frac{d^{|m|}}{d(\cos \theta)^{|m|}} P_{\ell}(\cos \theta)\right] e^{j m \phi} .
$$

For simplicity, we absorb all constants in $N_{\ell}^{m}$.

The essential observation is that the bracketed term is a polynomial in $x=\cos \theta$. At a bandwidth $L$, the largest spherical harmonic degree is $L-1$, so the largest power of $x$ is $L-1$ as well. Then we can rewrite the derivative term as the dot-product between the coefficient vector and a basis of monomials,

$$
\frac{d^{|m|}}{d(\cos \theta)^{|m|}} P_{\ell}(\cos \theta)=\mathbf{c}_{\ell m}^{T} \mathbf{x}_{m},
$$

where $\mathbf{x}_{m}=\left[x^{L-|m|-1}, x^{L-|m|-2}, \cdots, x, 1\right]^{T}, x=\cos \theta$ and $\mathbf{c}_{\ell m}$ contains the polynomial coefficients. Denoting further $u_{k m} \stackrel{\text { def }}{=}$ $\left(\sin \theta_{k}\right)^{|m|} e^{-j m \phi_{k}}$, we have

$$
f_{\ell}^{m}=N_{\ell}^{m} \mathbf{c}_{\ell m}^{T} \mathbf{X}_{m} \mathbf{A} \mathbf{u}_{m}
$$

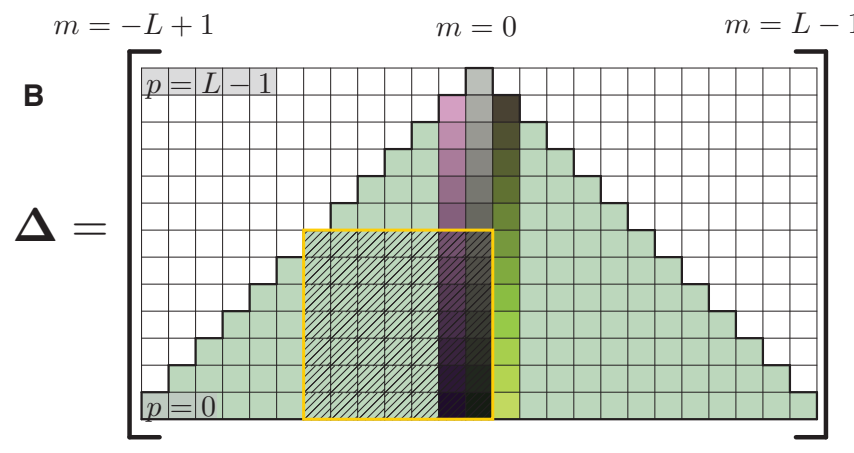

Fig. 1. Recoverable part of the data matrix $\Delta$ (shaded). Columns are indexed from left to right by $m,-(L-1) \leq m \leq(L-1)$, corresponding to spherical harmonic order. Rows are indexed bottom to top by $p, 0 \leq p \leq(L-1)$ corresponding to powers of $\cos \theta$. Note that the triangular part of the data matrix does not coincide with the spherical harmonic spectrum, although there is an invertible mapping between the two.

where $\mathbf{X}_{m}=\left[\begin{array}{lll}\mathbf{x}_{m, 1} & \cdots & \mathbf{x}_{m, K}\end{array}\right], \mathbf{A}=\operatorname{diag}\left(\alpha_{1}, \ldots, \alpha_{K}\right), \mathbf{u}_{m}=$ $\left[u_{1, m} \cdots u_{K, m}\right]^{T}$, and $\mathbf{x}_{m, k}$ is like $\mathbf{x}_{m}$, but for a particular $\theta=\theta_{k}$.

With this notation in hand, it is instructive to define the data matrix $\Delta$ as

$$
\boldsymbol{\Delta} \stackrel{\text { def }}{=} \mathbf{X}_{0} \mathbf{A U}
$$

where $\mathbf{U} \stackrel{\text { def }}{=}\left[\mathbf{u}_{(-L+1)}, \cdots, \mathbf{u}_{0}, \cdots, \mathbf{u}_{L-1}\right]$. Rows of $\boldsymbol{\Delta}$ correspond to decreasing powers of $\cos \theta_{k}$, and columns correspond to $u_{k m}$. Computing $f_{\ell}^{m}$ then amounts to applying a linear functional on $\boldsymbol{\Delta}$. This linear functional can be thought of as an inner product with a mask that is overlaid over $\Delta$. The support of the mask for $f_{\ell}^{m}$ is on the column corresponding to $m$, and on the rows corresponding to $\ell \geq|m|$. That means that certain parts of the data matrix are not involved in the creation of any spectral coefficient, and consequently, they cannot be recovered from the spectrum.

Element of $\boldsymbol{\Delta}$ at the position $(p, m)$ (with reference to Fig. 1) can be expanded as

$$
\sum_{k=1}^{K} \alpha_{k} x_{k}^{p}\left(\sin \theta_{k}\right)^{|m|} e^{-j m \phi_{k}}
$$

where $p$ varies from 0 to $L-1$, and $m$ from $-(L-1)$ to $(L-1)$. For either positive or negative $m$, the sum (12) is a sum of $2 \mathrm{D}$ exponentials. To make it obvious, for positive $m$ we can write it as $\sum_{k} \alpha_{k} x_{k}^{p} u_{k 1}^{m}$. If $\mathbf{U}$ was Vandermonde, and if we could retrieve the whole $\Delta$, our problem would correspond to a problem of 2D harmonic retrieval [19]. However, 1) $\mathbf{U}$ is only almost Vandermonde - it is a concatenation of two Vandermonde matrices due to the absolute value in the exponent of $u_{k m}, 2$ ) we can only recover a triangular part of $\boldsymbol{\Delta}$, illustrated in Fig. 1. Accordingly, we devise a different scheme to retrieve the signal parameters, achieving significantly better sampling efficiency than adapting the results on harmonic retrieval.

Define next $\mathbf{f}_{m}$ as the vector containing all spectral coefficients of order $m$,

$$
\mathbf{f}_{m}=\left[f_{|m|}^{m}, f_{|m|+1}^{m}, \ldots, f_{L}^{m}\right]^{T},
$$

and let $\mathbf{C}_{m}$ be the matrix created by stacking $N_{\ell}^{m} \mathbf{c}_{\ell m}^{T}$ for all admissible $\ell$,

$$
\mathbf{C}_{m}=\left[N_{|m|}^{m} \mathbf{c}_{|m|, m}, N_{|m|+1}^{m} \mathbf{c}_{|m|+1, m}, \ldots, N_{L-1}^{m} \mathbf{c}_{L-1, m}\right]^{T} .
$$


It follows that

$$
\mathbf{f}_{m}=\mathbf{C}_{m} \mathbf{X}_{m} \mathbf{A} \mathbf{u}_{m} .
$$

Recall that the largest power of any $x_{k}$ in $\mathbf{X}_{m}$ is $(L-|m|-1)$. In order to proceed, we need the following lemma.

\section{Lemma 1. The coefficient matrices $\mathbf{C}_{m}$ are invertible for every $m$.}

Thus we can recover $\mathbf{d}_{m} \stackrel{\text { def }}{=} \mathbf{X}_{m} \mathbf{A} \mathbf{u}_{m}$ from $\mathbf{f}_{m}$ as $\mathbf{d}_{m}=$ $\mathbf{C}_{m}^{-1} \mathbf{f}_{m}$. The vector $\mathbf{d}_{m}$ is a linear combination of the columns of $\mathbf{X}_{m}$, i.e. it is a linear combination of exponentials. It is exactly the recoverable part of column $m$ of the data matrix $\boldsymbol{\Delta}$ (see Fig. 1). Same as in the Euclidean case, we search for a finite impulse response filter that annihilates $\mathbf{d}_{m}$, and then find $\left\{x_{k}\right\}_{k=1}^{K}$ as the roots of the annihilating filter.

Consider a finite impulse response filter $H(z)$ with the transfer function $H(z) \stackrel{\text { def }}{=} \prod_{k=1}^{K}\left(1-x_{k} z^{-1}\right) \stackrel{\text { def }}{=} \sum_{n=0}^{K} h_{n} z^{-n}$, where $\mathbf{h}=$ $\left[1, h_{1}, \ldots, h_{K}\right]^{T}$ is the vector of filter coefficients. The response of $H(z)$ to a signal of the form $y_{n}=\sum_{k=1}^{K} b_{k} x_{k}^{n}$ is

$$
(y * h)_{n}=\sum_{m=0}^{K} y_{n-m} h_{m}=x_{k}^{n} \sum_{k=1}^{K} b_{k} \sum_{m=0}^{K} h_{m} x_{k}^{-m}=0,
$$

so $H(z)$ annihilates linear combinations of exponentials.

In order to find $H(z)$, we observe that the annihilation relation (16) states that the filter coefficients $\mathbf{h}$ are orthogonal to any length$(K+1)$ segment of any linear combination of exponentials $x_{k}^{n}$. If we can find enough length- $(K+1)$ segments of some linear combinations of the sought exponentials, we could find $\mathbf{h}$ as the orthogonal complement of the subspace spanned by these segments.

In the spherical case, we have that $\mathbf{d}_{m}$ is a linear combination of the sought exponentials for every admissible $m$. As the required length of the segment is $K+1$, we can only use those $m$ s such that the length of $\mathbf{d}_{m}$ is at least $K+1$. We could get the required number of samples from $\mathbf{d}_{0}$ by setting $L=2 K+1$, but this strategy ignores spectral coefficients for $m \neq 0$. Referring to Fig. 1, it is clear that we can get the rows of the annihilation matrix from any column ( $m=$ const.) of sufficient length.

In summary, every $\mathbf{d}_{m}$ is a linear combination of exponentials, and we want to compute the bases of these exponentials. If we can observe enough linear combinations of sufficient length, we can construct a matrix (denoted $\mathbf{Z}$ in Algorithm 1) with $K$ independent rows, whose nullspace contains the annihilation filter. The cosines of the spherical colatitudes-bases of exponentials annihilated by the annihilation filter-are obtained by factoring $H(z)$ into first order terms. We show in [16] that the described construction indeed ensures that $\mathbf{Z}$ have $K$ independent rows.

In order to recover the azimuths $\left(\phi_{k}\right)_{k=1}^{K}$, note that after recovering the colatitudes, we can construct the matrices $\mathbf{X}_{m}$. Furthermore, note that for $|m| \leq L-K, \mathbf{C}_{m} \mathbf{X}_{m}$ has full column rank, as a product of two full rank matrices. Thus we can compute $\mathbf{A} \mathbf{u}^{(m)}$, and the azimuths $\left(\phi_{k}\right)_{k=1}^{K}$ as the phase difference between $\mathbf{A} \mathbf{u}_{0}$ and $\mathbf{A} \mathbf{u}_{1}$.

For our techniques to be applicable, we need to ensure that all $\theta_{k}$ and $\phi_{k}$ be distinct. As in applications this may be violated, we observe that for a finite collection of spikes, there will always exist a choice of the coordinate system such that this is satisfied. We exploit this fact, formalized in the following lemma.

Lemma 2. Consider a collection of Dirac delta functions on the sphere, $f(\omega)=\sum_{k=1}^{K} \alpha_{k} \delta\left(\omega ; \omega_{k}\right)$, and a random rotation $\mathbf{R}$ drawn uniformly from the $3 D$ rotation group $\mathbb{S O}_{3}$. Then almost surely, $\mathbf{R} f$ contains Diracs with distinct $\theta_{k}$ and distinct $\phi_{k}$.
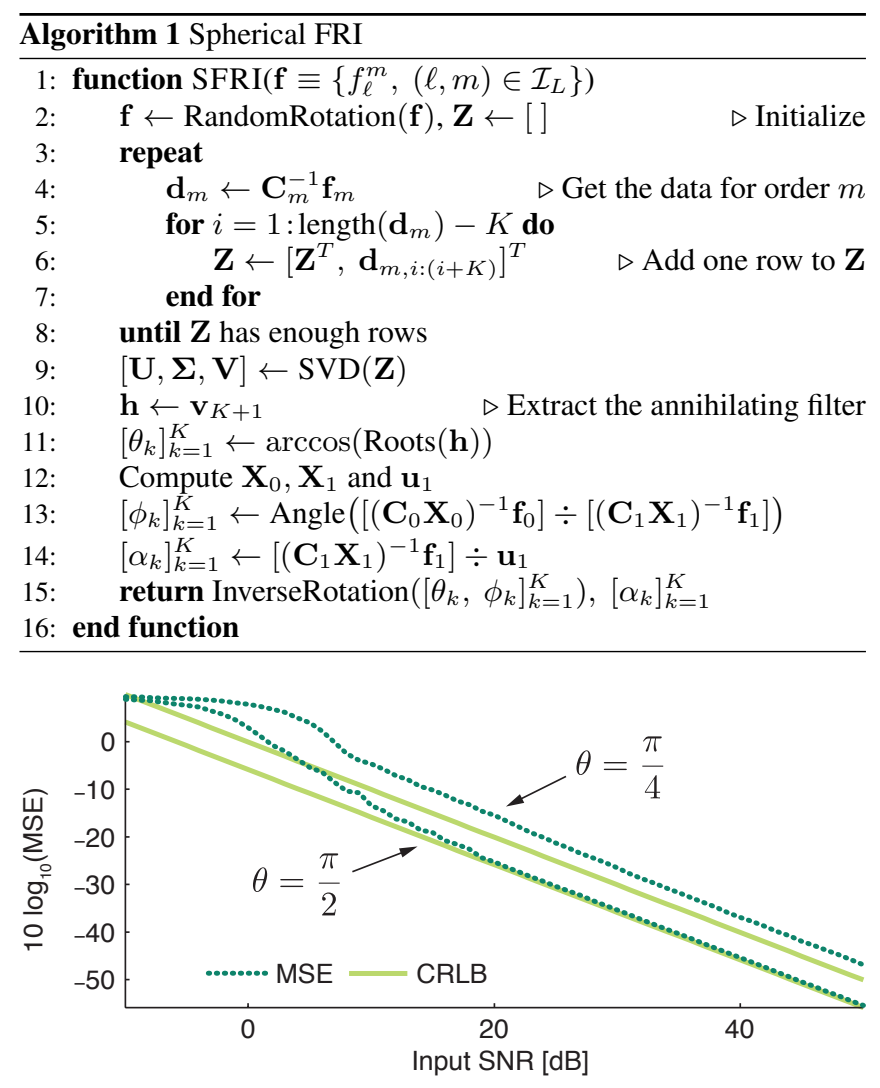

Fig. 2. Comparison between the mean squared error (MSE) in estimating the spherical location $(\theta, \phi)$, with $K=1$, and the CramérRao lower bound, at two different colatitudes.

Because we can rotate a spherical function by applying a linear operator on its spectrum, we can apply a random rotation before estimating the signal parameters, and then inverse rotate after estimation. The described procedure is summarized in Algorithm 1. Collecting the above observations, we state our main result.

Theorem 1. Let $f$ be a bandlimited observation of a weighted sum of $K$ Diracs on the sphere. Algorithm 1 perfectly recovers $f$ from $\lceil K+\sqrt{K}\rceil^{2}$ spherical samples.

There is a different way to look at this result. Unlike in the temporal Euclidean case, usually we do not get to choose $L$. It is useful to know how many Diracs we can reconstruct with a fixed sampling kernel. By solving for $K$ we get

$$
K \leq\left\lfloor\left(L-\left(L+\frac{1}{4}\right)^{1 / 2}+\frac{1}{2}\right)\right\rfloor .
$$

For the same number of Diracs, the algorithm of DeslauriersGauthier and Marziliano [15] requires approximately four times more samples.

\subsection{Estimation in the Presence of Noise}

Theorem 1 and Algorithm 1 provide a tool to recover sparse signals on the sphere in the noiseless case. In the presence of noise $\mathbf{Z}$ will generally not have a nullspace. A simple approach is to use the right singular vector corresponding to the smallest singular value. Let $\mathbf{Z}=\mathbf{U} \boldsymbol{\Sigma} \mathbf{V}^{H}$ be the SVD of $\mathbf{Z}$. Then we can set $\mathbf{h}=\mathbf{v}_{K+1}$. 

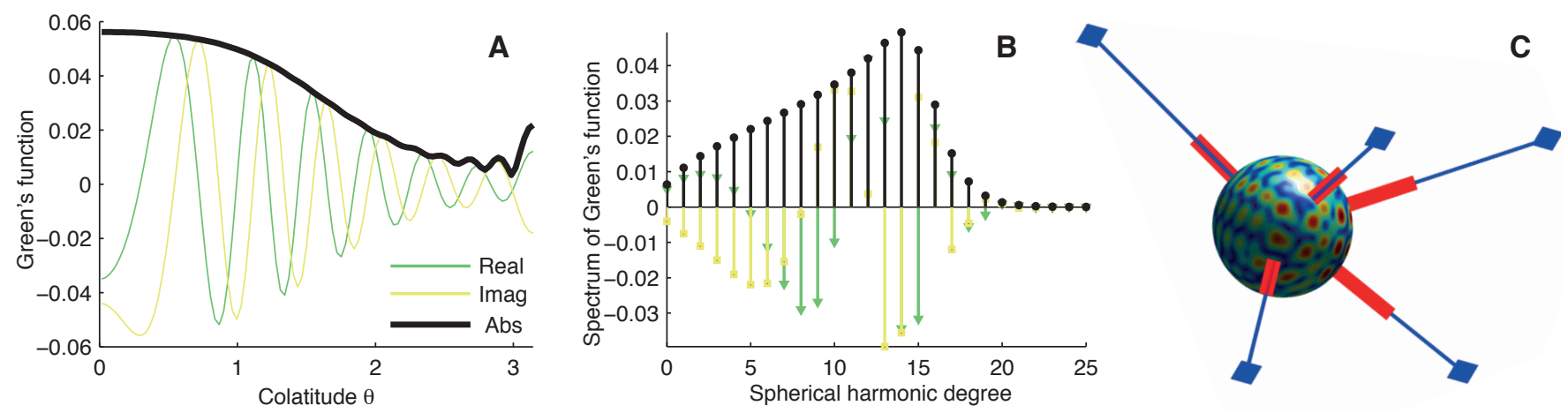

Fig. 3. Multiple DOA estimation by a spherical microphone array at $f=4000 \mathrm{~Hz}$, with $r=0.2 \mathrm{~m}$. (A) Real part, imaginary part and absolute value of the Green's function. (B) Real part, imaginary part and absolute value of the spectrum. (C) Simulation results for $K=2$. Blue diamonds represent the source locations, and thick red lines show the estimated directions. Size of the sphere is exaggerated for the purpose of illustration. The sphere color corresponds to the absolute value of the measured function, induced on the sphere by the sources.

Fig. 2 shows the variance of our estimator compared with the Cramér-Rao lower bound for $K=1$. The computation of the bound is given in [16].

\section{APPLICATION: SOUND SOURCE LOCALIZATION}

In this section, we address the estimation of the directions-of-arrival (DOA) of multiple narrowband sound sources using a spherical microphone array. In the wideband case, appropriately combining the estimates at different frequencies could eventually only improve the DOA estimates.

We assume that the microphones are distributed on the surface of a sphere, either open or rigid. Therefore, the microphone signals represent samples of a time-varying function on $\mathbb{S}^{2}$. If a sound source emits a sinusoid, every microphone measures a complex numberthe amplitude and the phase of that sinusoid shaped by the characteristics of the propagating medium and of the spherical array casing.

Suppose that a source of unit intensity is located at $\mathbf{r}_{s}$, and that the microphones are mounted on a rigid sphere of radius $r$ with center at the origin. The response measured by the microphone at $\mathbf{r}$, such that $\|\mathbf{r}\|=r$, is given by the corresponding Green's function. For a wavenumber $k=2 \pi f / c$, where $f$ is the frequency and $c$ is the speed of sound, the Green's function can be shown to be [7],

$$
g\left(\mathbf{r} \mid \mathbf{r}_{s}, k\right)=\frac{j k}{4 \pi} \sum_{\ell=0}^{\infty} b_{\ell}(k r) h_{\ell}^{(1)}\left(k r_{s}\right)(2 \ell+1) P_{\ell}\left(\left\langle\hat{\mathbf{r}}, \hat{\mathbf{r}}_{s}\right\rangle\right),
$$

where $h_{\ell}^{(1)}$ is the spherical Hankel function of the first kind and of order $\ell$, and $b_{\ell}(k r)$ is the mode strength [7].

The Green's function $g$ should be seen as a filter that describes how the point source's influence spreads over the sphere. Green's function is shown in Fig. 3(A), and its spectrum in Fig. 3(B).

For $K$ sound sources at locations $\left\{\mathbf{s}_{i}\right\}_{i=1}^{K}$, with complex intensities $\left\{\alpha_{i}\right\}_{i=1}^{K}$, a microphone at $\mathbf{r}$ measures

$$
f(\mathbf{r})=\sum_{i=1}^{K} \alpha_{i} g\left(\mathbf{r} \mid \mathbf{s}_{i}, k\right)
$$

If all $\left\|\mathbf{s}_{k}\right\|$ were equal, then (19) would correspond to a weighted sum of $K$ rotations of a known kernel. As it is unrealistic to assume that the sources are all at the same distance, we could hope that the shape of $g\left(\mathbf{r} \mid \mathbf{r}_{s}, k\right)$ does not change with $\mathbf{r}_{s}$. The shape is indeed preserved, although only approximately. Thus, we suppress the dependence on $\left\|\mathbf{r}_{s}\right\|$ and approximate as follows (setting $f(\omega)=f(\mathbf{r})$, where $\omega=(\theta, \phi)$ are the coordinates of $\mathbf{r})$,

$$
\begin{aligned}
f(\omega) & =\sum_{i=1}^{K} \alpha_{k} g\left(\omega \mid \mathbf{s}_{i}, k\right) \approx \sum_{i=1}^{K} \widetilde{\alpha}_{i} g\left(\omega \mid \omega_{\mathbf{s}, i}, k\right) \\
& =\left[\sum_{i=1}^{K} \widetilde{\alpha}_{i} \delta\left(\omega ; \omega_{\mathbf{s}, i}\right)\right] * g\left(\omega \mid \omega_{\mathbf{n}}, k\right) .
\end{aligned}
$$

Here, we absorbed $\alpha_{i}$ and additional (complex) scaling due to different distances into $\widetilde{\alpha}_{i}$. Angular coordinates of the north pole are denoted by $\omega_{\mathbf{n}}$, and $g\left(\omega \mid \omega_{\mathbf{n}}, k\right)$ is computed at some predefined $a v$ erage distance.

We thus reduced the sound source localization problem to a problem of finding parameters of a weighted sum of Diracs. In order to apply our spherical FRI algorithm, we need to verify that $g$ is bandlimited on the sphere. Fig. 3(B) shows that this is indeed the case. The bandwidth depends on frequency and on the sphere radius. Fig. 3(C) shows an example of recovering 5 sources at 4000 $\mathrm{Hz}$. Our intention is to highlight the usefulness of spherical finite rate of innovation sampling. Detailed comparisons with other DOA estimation algorithms are out of this paper's scope.

\section{CONCLUSION}

We presented a new state-of-the-art sampling theorem for FRI signals on the sphere. A multitude of signals are well-modeled as sparse signals on the sphere, and this motivates the development of the corresponding sampling theorems. We demonstrated this for sound source localization, but there are other examples such as the square kilometer array (SKA) [20].

An important issue not discussed in this paper is the denoising of the annihilation matrix. In the Euclidean case, this matrix is Toeplitz, and we can use alternating denoising techniques such as the Cadzow denoising algorithm [21]. On the sphere things seem more challenging. This is the topic of ongoing research. 


\section{REFERENCES}

[1] K. F. Evans, “The Spherical Harmonics Discrete Ordinate Method for Three-Dimensional Atmospheric Radiative Transfer," J. Atmos. Sci., vol. 55, no. 3, pp. 429-446, 1998.

[2] F. J. Simons, F. A. Dahlen, and M. A. Wieczorek, "Spatiospectral Concentration on a Sphere," SIAM Rev., vol. 48, no. 3, pp. 504-536, 2006.

[3] P. Audet, "Directional Wavelet Analysis on the Sphere: Application to Gravity and Topography of the Terrestrial Planets," $J$. Geophys. Res., vol. 116, no. E1, pp. 1-16, 2011.

[4] N. Jarosik, C. L. Bennett, J. Dunkley, B. Gold, M. R. Greason, M. Halpern, R. S. Hill, G. Hinshaw, A. Kogut, E. Komatsu, D. Larson, M. Limon, S. S. Meyer, M. R. Nolta, N. Odegard, L. Page, K. M. Smith, D. N. Spergel, G. S. Tucker, J. L. Weiland, E. Wollack, and E. L. Wright, "Seven-year Wilkinson Microwave Anisotropy Probe (WMAP) Observations: Sky Maps, Systematic Errors, and Basic Results," Astrophys. J. Suppl. Ser, vol. 192, no. 2, pp. 1-15, Feb. 2011.

[5] R. H. Macphie and E. H. Okongwu, "Spherical Harmonics and Earth-Rotation Synthesis in Radio Astronomy," IEEE Trans. Antennas Propag., vol. 23, no. 3, pp. 386-391, 1975.

[6] J. Meyer and G. Elko, “A Highly Scalable Spherical Microphone Array Based on an Orthonormal Decomposition of the Soundfield," in Proc. IEEE ICASSP, Orlando, FL, USA, 2002, pp. 1781-1784, IEEE.

[7] D. P. Jarrett, E. A. P. Habets, M. R. P. Thomas, and P. A. Naylor, "Rigid Sphere Room Impulse Response Simulation: Algorithm and Applications," J. Acoust. Soc. Am., vol. 132, no. 3, pp. 1462-1472, 2012.

[8] M. Vetterli, P. Marziliano, and T. Blu, "Sampling Signals with Finite Rate of Innovation," IEEE Trans. Signal Process., vol. 50, no. 6, pp. 1417-1428, June 2002.

[9] I. Maravic and M. Vetterli, "Sampling and Reconstruction of Signals With Finite Rate of Innovation in the Presence of Noise," IEEE Trans. Signal Process., vol. 53, no. 8, pp. 27882805, Aug. 2005.

[10] P. L. Dragotti and F. Homann, "Sampling Signals with Finite Rate of Innovation in the Presence of Noise," in Proc. IEEE ICASSP, Taipei, Taiwan, 2009, pp. 2941-2944, IEEE.
[11] I. Maravic and M. Vetterli, "Exact Sampling Results for Some Classes of Parametric Nonbandlimited 2-D Signals," IEEE Trans. Signal Process., vol. 52, no. 1, pp. 175-189, Jan. 2004.

[12] P. Shukla and P. L. Dragotti, "Sampling Schemes for Multidimensional Signals With Finite Rate of Innovation," IEEE Trans. Signal Process., vol. 55, no. 7, pp. 3670-3686, 2007.

[13] J. D. McEwen and Y. Wiaux, "A Novel Sampling Theorem on the Sphere," IEEE Trans. Signal Process., vol. 59, no. 12, pp. 5876-5887, 2011.

[14] Z. Khalid, R. A. Kennedy, and J. D. McEwen, “An OptimalDimensionality Sampling Scheme on the Sphere With Fast Spherical Harmonic Transforms," IEEE Trans. Signal Process., vol. 62, no. 17, pp. 4597-4610, 2014.

[15] S. Deslauriers-Gauthier and P. Marziliano, "Sampling Signals With a Finite Rate of Innovation on the Sphere," IEEE Trans. Signal Process., vol. 61, no. 18, pp. 4552-4561, 2013.

[16] I. Dokmanic and Y. M. Lu, "Sampling Sparse Signals on the Sphere: Algorithms and Applications," submitted to IEEE Trans. Sig. Process., 2015.

[17] J. R. Driscoll and D. M. Healy, "Computing Fourier Transforms and Convolutions on the 2-Sphere," Adv. Appl. Math., vol. 15, no. 2, pp. 202-250, June 1994.

[18] D. Duffy, Green's Functions with Applications, Chapman and Hall/CRC, 2001.

[19] F. Vanpoucke, M. Moonen, and Y. Berthoumieu, "An Efficient Subspace Algorithm for 2-D Harmonic Retrieval," in Proc. IEEE ICASSP, Adelaide, SA, Australia, 1994, pp. 461-464, IEEE.

[20] P. E. Dewdney, P. J. Hall, R. T. Schilizzi, and T. J. L. W. Lazio, "The Square Kilometre Array," Proc. IEEE, vol. 97, no. 8, pp. 1482-1496, June 2009.

[21] J. A. Cadzow, "Signal Enhancement-A Composite Property Mapping Algorithm," IEEE Trans. Acoust., Speech, Signal Process., vol. 36, no. 1, pp. 49-62, 1988. 